\title{
OPEN Clinical pattern of failure after a durable response to immune checkpoint inhibitors in non-small cell lung cancer patients
}

\author{
Ja Yoon $\mathrm{Heo}^{1,4}$, Shin Hye Yoo ${ }^{1}$, Koung Jin Suh ${ }^{2}$, Se Hyun Kim ${ }^{2 凶}$, Yu Jung Kim², \\ Chan-Young Ock ${ }^{1}$, Miso Kim ${ }^{1}$, Bhumsuk Keam ${ }^{1,3}$, Tae Min Kim ${ }^{1,3}$, Dong-Wan Kim ${ }^{1,3}$, \\ Dae Seog $\mathrm{Heo}^{1,3}$ \& Jong Seok Lee ${ }^{2}$
}

Although immune checkpoint inhibitors (ICls) can induce durable responses in non-small-cell lung cancer (NSCLC) patients, a significant proportion of responders still experience progressive disease after a period of response. Limited data are available on the clinical patterns of acquired resistance (AR) to ICls. Clinical and radiologic data from 125 NSCLC patients treated with anti-PD-1 or PD-L1 antibodies between 2011 and 2018 at two tertiary academic institutions were retrospectively reviewed. Overall, $63(50.4 \%)$ patients experienced AR after ICI treatment in a median of 10.7 months. Among the 13 patients with a partial response with $\mathrm{ICl}, 12$ (32.4\%) had only lymph node progression. Most patients ( $n=52,82.5 \%$ ) had one or two sites with progression (oligo-progression). The median overall survival (OS) after progression was significantly longer in the extrathoracic group than in the thoracic and liver progression groups (30.2 months [95\% confidence interval $(\mathrm{Cl}), 13.4$ to not reached (NR)], 11.7 months [95\% Cl, 9.5-21.1], and 5.4 months [95\% Cl, 2.6-NR], respectively, $P<0.001$ ). Patients with oligo-progression had significantly longer OS after AR than did the multi-progression patients (18.9 months [ $95 \% \mathrm{Cl}, 10.6-\mathrm{NR}$ ] vs. 8.8 months [ $95 \% \mathrm{Cl}, 5.7-\mathrm{NR}$ ], $P=0.04$ ). No significant difference in progression-free survival was observed between the subsequent chemotherapy and the $\mathrm{ICl}$ after AR groups $(P=0.723)$. Patients with $\mathrm{AR}$ after $\mathrm{ICl}$ treatment had a unique progression pattern with oligo-progression and high rates of progression only in the lymph nodes. Local treatment and/or continuation of ICls beyond AR might be an effective option.

Immune checkpoint inhibitors (ICIs), including anti- programmed death 1 (PD-1) or programmed death ligand 1 (PD-L1) antibodies, represent a breakthrough in the treatment of non-small-cell lung cancer (NSCLC). Previous studies have shown that high levels of PD-L1 expression on tumors and/or immune cells by immunohistochemistry are associated with improved clinical efficacy when compared with low levels or negative PD-L1 $1^{1-6}$. In patients with pretreated advanced NSCLC, ICI monotherapy resulted in durable clinical responses lasting more than 6 months and enabled patients to achieve 5-year overall survival (OS) without subsequent therapy and disease progression ${ }^{6-8}$. However, a significant proportion of long-term responders still experience progressive disease after a period of response time.

Acquired resistance (AR) refers to a clinical scenario in which cancer initially responds to immunotherapy, but it continues to grow and metastasize to other organs after a period of time. In the epidermal growth factor receptor (EGFR) mutant NSCLC, it is well-known that acquired mutations, including T790M, confer AR to tumors after EGFR tyrosine kinase inhibitor (TKI) treatment ${ }^{9}$. However, among patients with ICI treatment, more efforts are needed to elucidate which patients will experience AR and how tumor evolution or changes in the immune system lead to AR development. Defects in interferon-receptor signaling and antigen presentation pathways, tumor-associated neoantigen loss, alternate immune checkpoints, and alterations in the tumor microenvironment are believed to be involved in AR mechanisms ${ }^{10-12}$.

\footnotetext{
${ }^{1}$ Department of Internal Medicine, Seoul National University Hospital, Seoul, Republic of Korea. ${ }^{2}$ Department of Internal Medicine, Bundang Hospital, Seoul National University College of Medicine, 82, Gumi-ro 173 beon-gil, Bundang-Gu, Seongnam, Gyeonggi-do 13620, Republic of Korea. ${ }^{3}$ Cancer Research Institute, Seoul National University, Seoul, Republic of Korea. ${ }^{4}$ Present address: Department of Internal Medicine, National Health Insurance Service Ilsan Hospital, Goyang, Republic of Korea. ${ }^{\varpi}$ email: sehyunkim@snubh.org
} 
Recently, Gettinger et al. ${ }^{13}$ presented an impressive single-institution case series of 26 NSCLC patients who experienced AR of the PD-1 blockade. The authors reported the lymph node (LN) as the most common site of AR; their study included 11 cases with only LN progression. The majority of the included patients (88\%) showed progression in one or two sites of disease. Overall, 15 (58\%) patients received local therapy to sites of AR, while $11(42 \%)$ patients continued immunotherapy beyond progression ${ }^{14}$. To date, clinical data regarding subsequent treatment strategies and survival according to progression patterns after AR to ICI in NSCLC patients is sparse, especially for those with durable responses.

Therefore, in this study, we conducted a retrospective analysis to investigate the clinical patterns of AR after a durable response to ICIs in NSCLC patients. Additionally, we determined the implications for subsequent therapy in these patients.

\section{Results}

Patient characteristics. Of the 125 patients with a durable clinical benefit more than 6 months, 63 (50.4\%) patients with AR to ICIs were identified. The baseline clinicopathological characteristics of these patients are summarized in Table 1 . The majority $(\mathrm{n}=47,74.6 \%)$ of the patients were male, and just over half of the patients were pathologically diagnosed with adenocarcinoma $(n=35,55.5 \%)$. The majority of patients received ICI as first or second-line treatment for metastatic NSCLC $(n=47,74.6 \%) ; 21(33.3 \%)$ and $26(41.3 \%)$ patients received it as first and second-line treatment, respectively. The study included $15(23.8 \%)$ patients with driver mutations. Fifty-two $(82.5 \%)$ patients received monotherapy, while $11(17.5 \%)$ patients received combination therapy, including five (7.9\%) patients with PD-1 and CTLA-4 inhibitors, five (7.9\%) patients with PD-L1 and CTLA-4 inhibitors, and one (1.6\%) patient with a PD-1 inhibitor and chemotherapy. The median duration of follow-up was 18 months (range, 8-60) and 23 (36.5\%) patients were alive at the time of data analysis in December 2018.

Clinical patterns of ICI failure. Among all the included patients, the median time to AR was 10.7 months. The clinical patterns of AR after ICI treatment are summarized in Table 2 and shown based on their best objective response in Fig. 1 . The most common site of progressive disease was the lungs $(38 / 63,60.3 \%)$, followed by the LNs $(31 / 63,49.2 \%)$, and the brain $(6 / 63,9.5 \%)$. One of fifth $(14 / 63,22.2 \%)$ of patients had only LN progression at the time of AR. Twelve (85.7\%) of the 14 patients with LN progression only had achieved PR as their best response, while $2(14.3 \%)$ of the 14 patients achieved SD during ICI treatment $(P=0.04)$.

Next, we evaluated the pattern of failure according to the type of ICI treatment. Less frequent lung progression was observed in patients treated with ICI as first- or second-line treatment than in patients treated with ICI as at least third-line treatment $(48.9 \%$ vs. $93.8 \%$; $P$ value $=0.039)$. Progressive disease only in the LNs was observed among the patients who received first- or second line-treatment. There were no statistically significant differences between the two groups regarding the number of progression sites, previous best response, and the pre-existence of progression sites.

The clinical patterns of AR based on the number of progression sites are summarized in Table 3. Fifty-two $(82.5 \%)$ patients had progression in one or two lesions (oligo-progression group), while 11 (25.4\%) patients had progression in three or more lesions (multi-progression group). The lungs were the most frequent sites of progression in both groups. Progression of new lesions only was observed among $12(23.1 \%)$ and 0 (0\%) patients with oligo-progression and multi-progression, respectively $(P<0.001)$. Progression in the LNs only was also more frequent in patients with oligo-progression compared to patients with multi-progression $(27.0 \% \mathrm{vs.} 0 \%, P=0.103)$.

Survival differences based on the progression patterns. The median OS after AR was 10.9 months. OS after AR was analyzed according to the pattern of progression. When the patients were classified into subgroups (i.e. those who had intrathoracic, extrathoracic [excluding the liver], and liver progression), the median OS after progression was significantly longer in the extrathoracic subgroup than in the thoracic and liver progression subgroups (30.2 months [95\% CI, 13.4 to not reached (NR)], 11.7 months [95\% CI, 9.5-21.1], and 5.4 months [95\% CI, 2.6-NR], respectively, $P<0.001$, Fig. 2A). The median OS after progression was significantly longer in the oligo-progression group compared to that in the multiple progression group (18.9 months [ $95 \%$ CI, 10.6-NR] vs. 8.8 months [95\% CI, $5.7-\mathrm{NR}$ ], $P=0.04$, Fig. $2 \mathrm{~B}$ ). The progression patterns were not statistically significant when classified by progression in new lesions (Fig. 2C) nor nodal progression (Fig. 2D).

Subsequent therapy after AR. Among the 63 patients with AR to ICIs, 52 (82.5\%) patients received subsequent therapy after progression. Eleven (21.2\%) patients received local treatment, including radiotherapy and surgical resection. Forty-one (78.8\%) patients underwent systemic therapy after progression, including 25 $(48.1 \%)$ and $16(30.8 \%)$ patients who received cytotoxic chemotherapy and ICI after progression (Table 4). The median OS of the local therapy, chemotherapy, and ICI groups was 27.4, 23.2, and 29.1 months, respectively; there was no significant difference in survival between these groups $(P=0.787$, Fig. 3A). Additionally, there was no significant difference in the PFS between the chemotherapy and the ICI groups $(P=0.723$, Fig. 3B).

\section{Discussion}

In this study, after a median time of 10.4 months, patients who had a durable clinical benefit experienced AR. The majority of these patients $(52 / 63,82.5 \%)$ had progressive disease in one or two sites. Progression in the LNs only was observed in $22.2 \%$ patients at the time of AR; such patients received ICI as first or second-line treatment. Most $(12 / 14,85.7 \%)$ patients with progression in the LNs only had objective tumor shrinkage, partial response according to RECIST, during ICI treatment. Among patients with oligo-progression $(n=52)$, progression of the LNs only (27.0\%) or appearance of new lesions without evident progression of pre-existing lesions (23.1\%) was observed. The patterns of progression observed in this cohort of patients with NSCLC at the time of AR are very 


\begin{tabular}{|c|c|}
\hline & $\mathrm{N}=63$ \\
\hline \multicolumn{2}{|l|}{ Age (years) } \\
\hline Median (range) & $64(34-84)$ \\
\hline \multicolumn{2}{|l|}{ Sex } \\
\hline Male & 47 (74.6) \\
\hline Female & $16(25.4)$ \\
\hline \multicolumn{2}{|l|}{ Ethnicity } \\
\hline Asian & $63(100.0)$ \\
\hline \multicolumn{2}{|l|}{ Smoking } \\
\hline Never smoked & $21(33.3)$ \\
\hline Current or ex-smoker & $42(66.7)$ \\
\hline \multicolumn{2}{|l|}{ Pathology } \\
\hline Adenocarcinoma & $35(55.5)$ \\
\hline Poorly differentiated & $4(6.3)$ \\
\hline Sarcomatoid & $3(4.8)$ \\
\hline Large cell neuroendocrine & $2(3.2)$ \\
\hline Squamous & $19(30.2)$ \\
\hline \multicolumn{2}{|l|}{ Driver mutation } \\
\hline EGFR & $11(17.4)$ \\
\hline KRAS & $2(3.2)$ \\
\hline ALK & $1(1.6)$ \\
\hline ROS1 & $1(1.6)$ \\
\hline Not identified & $48(76.2)$ \\
\hline \multicolumn{2}{|l|}{ ICI treatment line } \\
\hline 1 & $21(33.3)$ \\
\hline 2 & $26(41.3)$ \\
\hline$\geq 3$ & $16(25.4)$ \\
\hline \multicolumn{2}{|l|}{ ICI treatment } \\
\hline PD-1 inhibitor & $36(57.1)$ \\
\hline PD-L1 inhibitor & $16(25.4)$ \\
\hline PD-1 inhibitor + CTLA-4 inhibitor & $5(7.9)$ \\
\hline PD-L1 inhibitor + CTLA-4 inhibitor & $5(7.9)$ \\
\hline PD-1 inhibitor + chemotherapy & $1(1.6)$ \\
\hline \multicolumn{2}{|l|}{ PD-L1 expression } \\
\hline$\geq 50 \%$ & $18(28.6)$ \\
\hline $1-49 \%$ & $10(15.9)$ \\
\hline $0 \%$ & $11(17.5)$ \\
\hline Not available & $24(38.1)$ \\
\hline \multicolumn{2}{|l|}{ Best objective response } \\
\hline Stable disease & $26(41.3)$ \\
\hline Partial response & 37 (58.7) \\
\hline Complete response & $0(0)$ \\
\hline
\end{tabular}

Table 1. Patient characteristics. EGFR epidermal growth factor receptor, $A L K$ anaplastic lymphoma kinase, $P D-1$ programmed death 1, PD-L1 programmed death ligand 1, CTLA-4 Cytotoxic T-Lymphocyte Associated Protein $4 .{ }^{\star}$ Data are presented as the median (range) or number $(\%)$.

unique considering that most patients treated in the conventional chemotherapy era develop systemic progression including pre-existing diseases. Even after having AR, patients with oligo-progression could be treated with local ablative therapy or continued ICI beyond AR. In the survival analysis, there was significantly longer OS among the oligo-progressors compared to that among systemic or multiple site progressors. We observed no significant difference in terms of PFS and OS between patients with subsequent chemotherapy and those with continuation of ICI beyond progression.

A notable finding in our study was that a significant portion of patients with AR to ICIs had progression of the LNs only, with sustained anti-cancer efficacy of ICI in pre-existing lesions. Among patients with a PR response with ICI ( $\mathrm{n}=37,58.7 \%), 12(32.4 \%)$ patients had progression of the LNs only. In the previous study by Gettinger et al. it was also reported that $42 \%$ of NSCLC patients who achieved PR after ICI treatment had AR limited to the $\mathrm{LNs}^{11}$. The study also suggested that the malignant $\mathrm{LN}$ environment may lead to the immunosuppressive evolution of cells within the node. 


\begin{tabular}{|c|c|c|c|c|}
\hline & All patients & 1st/2nd line ICI & $\geq 3$ rd line ICI & \multirow[b]{2}{*}{ P-value } \\
\hline & $(n=63)$ & $(n=47)$ & $(n=16)$ & \\
\hline \multicolumn{5}{|l|}{ Previous best response } \\
\hline PR & $37(58.7)$ & $16(34.0)$ & $6(37.5)$ & 0.089 \\
\hline SD ( $\geq 6$ months) & $26(41.3)$ & $31(66.0)$ & $10(62.5)$ & \\
\hline \multicolumn{5}{|c|}{ Time to acquired resistance } \\
\hline Median (range) & $10.7(6.0-47.2)$ & $10.8(6.0-47.2)$ & $10.2(6.4-22.9)$ & 0.052 \\
\hline \multicolumn{5}{|c|}{\begin{tabular}{|l|} 
Overall survival after progression \\
\end{tabular}} \\
\hline Median (range) & $10.9(0.0-43.7)$ & $11.5(0.0-43.7)$ & $9.6(0.0-30.4)$ & 0.775 \\
\hline \multicolumn{5}{|c|}{ Number of progression sites } \\
\hline 1 & $46(73.0)$ & $36(76.6)$ & $10(62.5)$ & 0.553 \\
\hline 2 & $6(9.5)$ & $4(8.5)$ & $2(12.5)$ & \\
\hline$\geq 3$ & $11(17.5)$ & $7(14.9)$ & $4(25.0)$ & \\
\hline \multicolumn{5}{|l|}{ Progression site $^{\mathrm{a}}$} \\
\hline Lung & $38(60.3)$ & $23(48.9)$ & $15(93.8)$ & 0.004 \\
\hline \multicolumn{5}{|l|}{ Non-lung visceral organ } \\
\hline Liver & $4(6.3)$ & $2(4.3)$ & $2(12.5)$ & 1 \\
\hline Colon & $1(1.6)$ & $1(2.1)$ & $0(0)$ & \\
\hline Adrenal gland & $1(1.6)$ & $1(2.1)$ & $0(0)$ & \\
\hline Heart (pericardium) & $2(3.2)$ & $1(2.1)$ & $1(6.2)$ & \\
\hline Brain & $6(9.5)$ & $5(10.6)$ & $1(6.2)$ & \\
\hline Bone & $4(6.3)$ & $2(4.3)$ & $2(12.5)$ & 1 \\
\hline Lymph node & 31 & 24 & 7 & 0.265 \\
\hline Neck & $5(7.9)$ & $3(6.4)$ & $2(12.5)$ & 0.274 \\
\hline Axilla & $2(3.2)$ & $1(2.1)$ & $1(6.2)$ & \\
\hline Thoracic & $20(32.7)$ & $16(34.0)$ & $4(25.0)$ & \\
\hline Abdomen & $4(6.3)$ & $4(6.3)$ & $0(0)$ & \\
\hline \multicolumn{5}{|c|}{\begin{tabular}{|l|} 
Pre-existence of progression site \\
\end{tabular}} \\
\hline New only & $13(20.6)$ & $11(23.4)$ & $1(6.2)$ & 0.201 \\
\hline Existing only & $43(68.3)$ & $32(68.1)$ & $12(75.0)$ & \\
\hline Both new and existing & $7(11.1)$ & $4(8.5)$ & $3(18.8)$ & \\
\hline \multicolumn{5}{|c|}{\begin{tabular}{|l|} 
Pattern of progression sites \\
\end{tabular}} \\
\hline Lymph node only & $14(22.2)$ & $14(30.0))$ & $0(0)$ & 0.013 \\
\hline Extranodal organs & $49(77.8)$ & $33(70.0)$ & $16(100)$ & \\
\hline
\end{tabular}

Table 2. Clinical Patterns of the failure of immune checkpoint inhibitors. Bold values denote statistical significance at the $p<0.05$ level ICI immune checkpoint inhibitors, $P R$ partial response, $S D$ stable disease. a Patients were included in more than one group based on the site of progression.

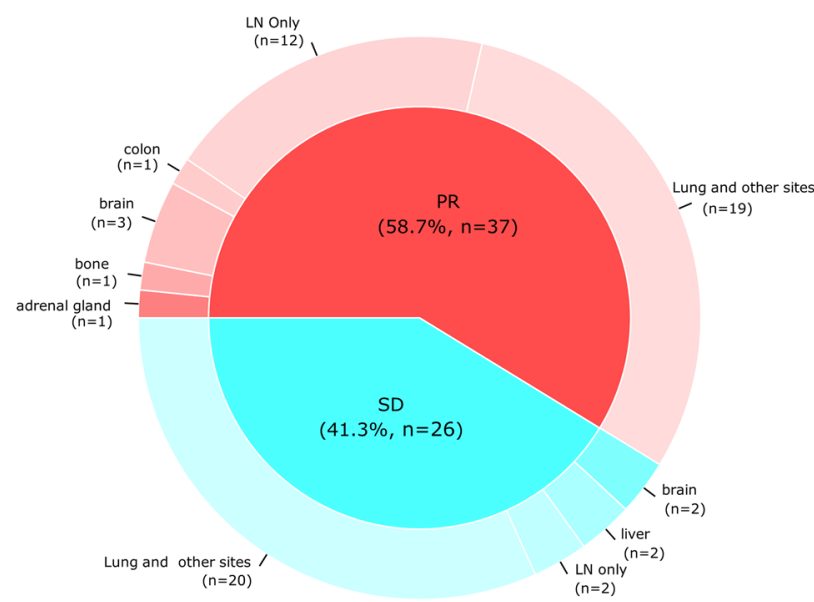

Figure 1. Donut plot showing sites of acquired resistance by best objective response to immune checkpoint inhibitors. $L N$ lymph node, $P R$ partial response, $S D$ stable disease. 


\begin{tabular}{|c|c|c|c|}
\hline & Oligo-progression & Multiple progression & \\
\hline & (Progression site 1-2) & (Progression site $3 \leq$ ) & \\
\hline & $(n=52)$ & $(\mathrm{n}=11)$ & P-value \\
\hline Age (years) & & & \\
\hline Median (range) & $64(34-84)$ & $62(49-74)$ & 0.752 \\
\hline Sex & & & \\
\hline Male & $38(73.1)$ & $9(81.8)$ & 0.714 \\
\hline Female & $14(26.9)$ & $2(18.2)$ & \\
\hline Smoking & & & \\
\hline Never smoked & $19(36.5)$ & $2(18.2)$ & 0.31 \\
\hline Current or ex-smoker & $33(63.5)$ & $9(81.8)$ & \\
\hline \begin{tabular}{|l|} 
Pathology \\
\end{tabular} & & & \\
\hline \begin{tabular}{|l|} 
Squamous \\
\end{tabular} & $16(30.8)$ & $3(27.3)$ & 0.822 \\
\hline Non-Squamous & $36(69.2)$ & $8(62.7)$ & \\
\hline ICI treatment line & & & \\
\hline 01-Feb & $40(76.9)$ & $7(63.6)$ & 0.449 \\
\hline $3 \leq$ & $12(23.1)$ & $4(36.4)$ & \\
\hline Monotherapy & & & \\
\hline PD-1 inhibitor & 27 (51.9) & $9(81.8)$ & \\
\hline PD-L1 inhibitor & $16(30.8)$ & $0(0)$ & \\
\hline Combination therapy & & & \\
\hline PD-1 inhibitor + chemotherapy & $1(1.9)$ & $0(0)$ & \\
\hline PD-1 inhibitor + CTLA-4 inhibitor & $3(5.8)$ & $2(18.2)$ & \\
\hline PD-L1 inhibitor + CTLA-4 inhibitor & $5(9.6)$ & $0(0.0)$ & \\
\hline Previous response & & & \\
\hline SD & $29(55.8)$ & $8(72.7)$ & 0.502 \\
\hline PR & $23(44.2)$ & $3(27.3)$ & \\
\hline Progression site $^{\mathrm{a}}$ & & & \\
\hline Lung & $28(53.8)$ & $10(90.9)$ & 0.039 \\
\hline Non-lung visceral organ & & & \\
\hline Liver & $2(3.8)$ & $2(18.2)$ & 0.275 \\
\hline Colon & $1(1.9)$ & $0(0)$ & \\
\hline Adrenal gland & $1(1.9)$ & $0(0)$ & \\
\hline Heart (pericardium) & $1(1.9)$ & $1(9.1)$ & \\
\hline Brain & $5(9.6)$ & $1(9.1)$ & 1 \\
\hline Bone & $1(1.9)$ & $3(27.3)$ & 0.015 \\
\hline Lymph node & $14(23.6)$ & $11(100)$ & $<0.001$ \\
\hline Neck & $3(5.8)$ & $1(9.1)$ & \\
\hline Axilla & $1(1.9)$ & $1(9.1)$ & \\
\hline Thoracic & $10(15.9)$ & $10(90.9)$ & \\
\hline Abdomen & $4(6.3)$ & $0(0)$ & \\
\hline \begin{tabular}{|l|} 
Pre-existence of progression site \\
\end{tabular} & & & \\
\hline New only & $12(23.1)$ & $0(0)$ & $<0.001$ \\
\hline Existing only & $39(75.0)$ & $5(45.5)$ & \\
\hline Both new and existing & $1(1.9)$ & $6(54.5)$ & \\
\hline Pattern of progression sites & & & \\
\hline Lymph node only & $14(27.0)$ & $0(0)$ & 0.103 \\
\hline Extranodal organs & $38(73.0)$ & $11(100.0)$ & \\
\hline
\end{tabular}

Table 3. Baseline characteristics based on oligo-progression. Bold values denote statistical significance at the $p<0.05$ level $P R$ partial response, $S D$ stable disease, $P D-1$ programmed death $1, P D-L 1$ programmed death

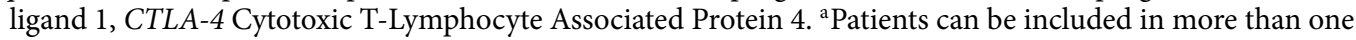
group based on the site of progression ${ }^{13}$.

It should be also noted that that patients with oligo-progression showed prolonged OS compared to those with multi-progression after AR in our study. Potential hypotheses for this finding might be the "the cancer-immunity cycle" 15 . Tumors in still "immune surveillance active" hosts are not able to proliferate or metastasize to other 
A
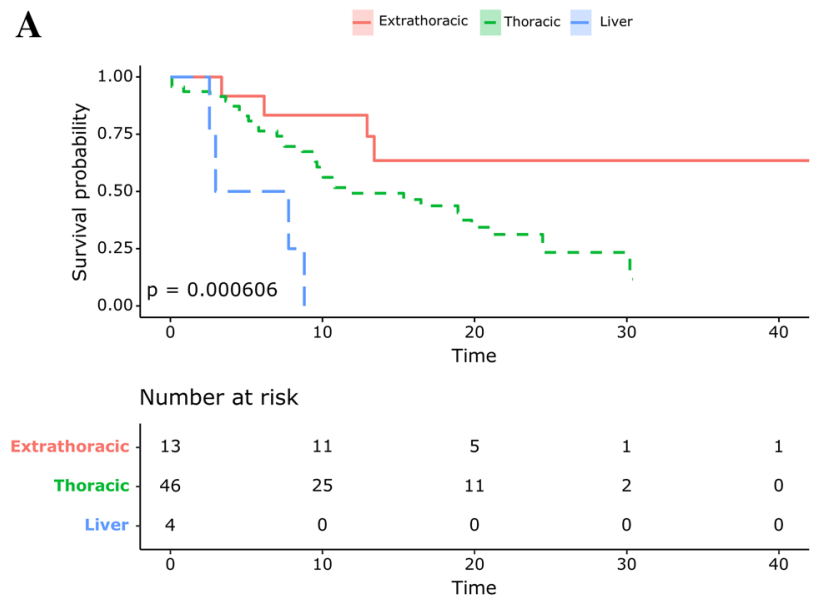

C
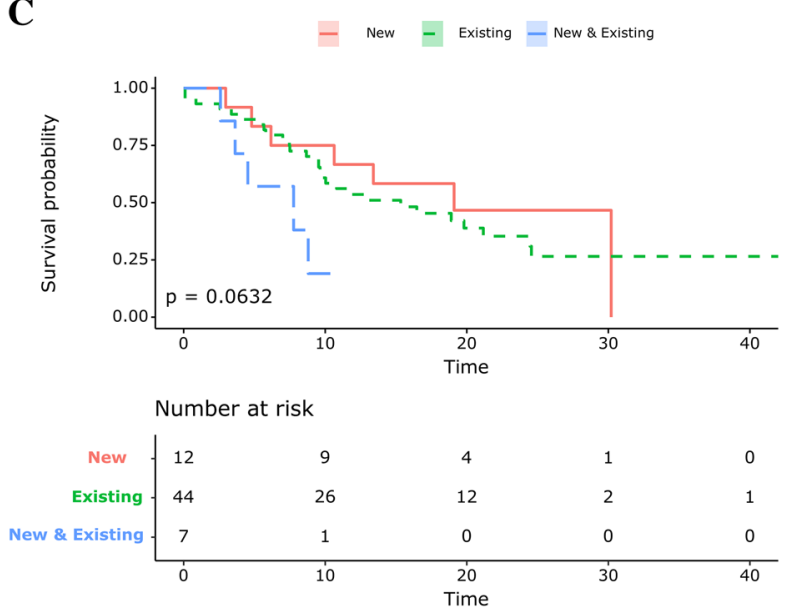

B

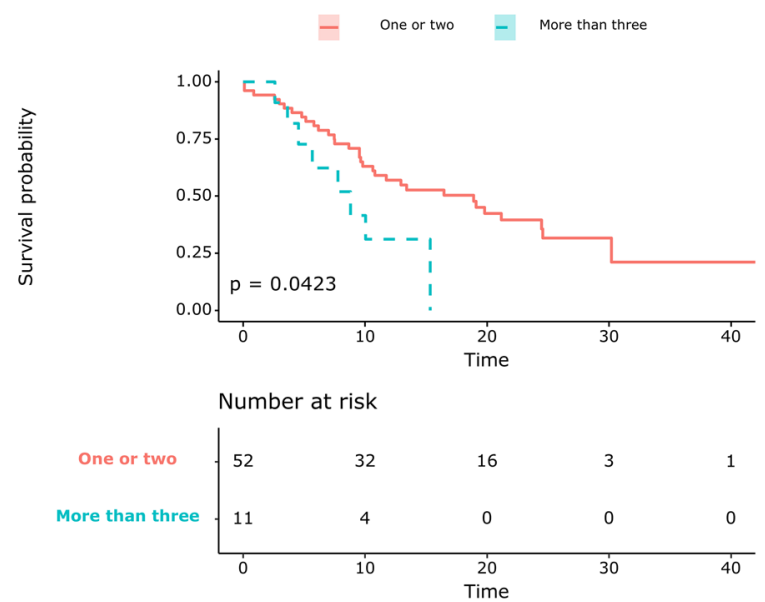

D

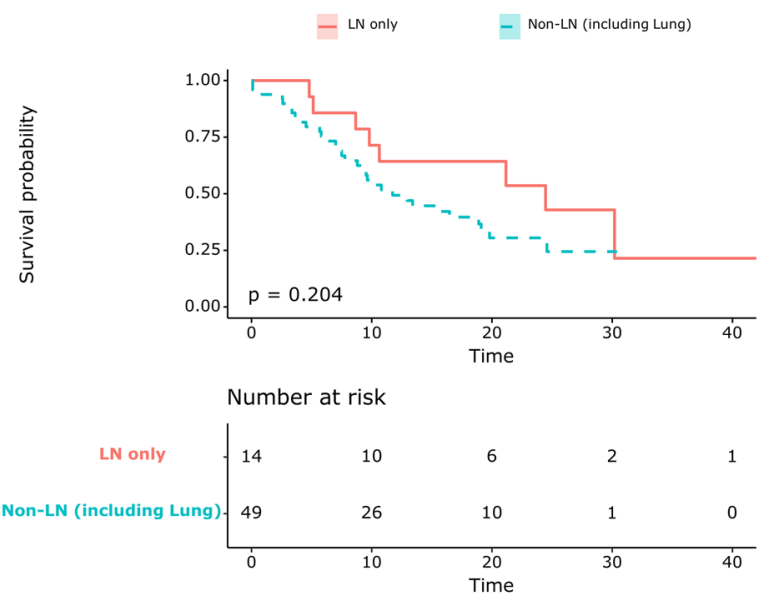

Figure 2. Kaplan-Meier survival curves showing overall survival from the point of acquired resistance for (A) the subgroup that had thoracic, extrathoracic, and liver progression only (B) the subgroup that had one or two progression sites or more than three progression sites $(\mathbf{C})$ the subgroup that had progression from new lesions, existing lesions, and both new and existing lesions (D) the subgroup that had only lymph node and extranodal progression (including the lung).

organs unless they obtain the novel phenotype of immune evasion. Therefore, it is easily conceivable that lung cancer clones in the progressive site might have evolved to escape host immune surveillance with either loss of heterozygosity in human leukocyte antigens or depletion of the expressed neoantigens ${ }^{16}$.

In this study, physicians preferred local therapy and immunotherapy beyond progression for patients with oligo-progression. For NSCLC, there was an interesting retrospective report from the phase III OAK study that atezolizumab showed acceptable efficacy and safety after progression with patients with good performance ${ }^{17}$. To date, ICI has been reported to show post-progression efficacies in a limited subset of patients including those with metastatic urothelial carcinoma, renal cell carcinoma and melanoma, and $\mathrm{NSCLC}^{18-22}$. However, whether treatment beyond progression will be effective is not yet elucidated in patients with AR. Gettinger et al. reported that 11 NSCLC patients continued immunotherapy beyond $\mathrm{AR}^{13}$. Adam LC et al. reported an AR case of successful local control with cryotherapy combined with immunotherapy beyond progression ${ }^{23}$. Further analysis will help to define the clinical benefit for NSCLC patients with a durable response treated with ICI beyond progression.

There are several limitations to this study. First, this was a retrospective analysis. As not many patients showed a durable response to ICI, only a small number of patients were included in the study, resulting in limited statistical power. Therefore, the small study size prohibited us from performing comparisons between the different subgroups. Second, the study population was very diverse, including not only patients with PD-1 inhibitor or PD-L1 inhibitor monotherapy, but also patients with combination therapy including cytotoxic drugs or CTLA-4 inhibitors. Third, although the PD-L1 expression profile is a very important prognostic factor, our study included the PD-L1 profile utilizing three different anti-PD-L1 antibodies (22C3, E1L3N, and SP263). As most of the patients $(81.0 \%)$ were involved in clinical trials, we were not able to ascertain the immunohistochemistry profiles for some patients, due to patient information protection regulations. Besides, information on co-medication such as antibiotics, corticosteroids, opioids, or proton pump inhibitors, which can affect ICI response, was not fully available. Lastly, there may be bias in the selection of subsequent therapy, as each individual physician was responsible for selecting the appropriate treatment. 


\begin{tabular}{|c|c|c|c|}
\hline & $\begin{array}{l}\text { All patients } \\
(\mathrm{n}=52)\end{array}$ & $\begin{array}{l}\text { 1st/2nd line ICI } \\
(n=40)\end{array}$ & $\begin{array}{l}\geq 3 \text { rd line ICI } \\
(n=12)\end{array}$ \\
\hline \multicolumn{4}{|l|}{ Local therapy $(n=11)$} \\
\hline Surgery & $2(3.8)$ & $2(5.0)$ & $0(0)$ \\
\hline Radiotherapy & $7(13.5)$ & $6(15.0)$ & $1(8.3)$ \\
\hline Gamma-knife surgery & $2(3.8)$ & $2(5.0))$ & $0(0)$ \\
\hline \multicolumn{4}{|c|}{ Systemic therapy $(n=41)$} \\
\hline Immunotherapy & $16(30.8)$ & $16(40.0)$ & $0(0.0)$ \\
\hline \multirow[t]{2}{*}{ Chemotherapy } & $25(48.1)$ & $16(40.0)$ & $9(75.0)$ \\
\hline & $\begin{array}{l}\text { All patients } \\
(\mathrm{n}=52)\end{array}$ & \begin{tabular}{|l|} 
Oligo-progression \\
(Progression site 1-2) \\
$(\mathrm{n}=43)$
\end{tabular} & $\begin{array}{l}\text { Multiple progression } \\
(\text { Progression site } 3 \leq) \\
(\mathbf{n}=9)\end{array}$ \\
\hline \multicolumn{4}{|l|}{ Local therapy $(n=11)$} \\
\hline Surgery & $2(3.8)$ & $2(4.7)$ & $0(0)$ \\
\hline Radiotherapy & $7(13.5)$ & $6(14.0)$ & $1(11.1)$ \\
\hline Gamma-knife surgery & $2(3.8)$ & $2(4.7)$ & $0(0)$ \\
\hline \multicolumn{4}{|c|}{ Systemic therapy $(n=41)$} \\
\hline Immunotherapy & $16(30.8)$ & $14(32.6)$ & $2(22.2)$ \\
\hline chemotherapy & $25(48.1)$ & $19(44.2)$ & $6(66.7)$ \\
\hline \multirow[t]{6}{*}{ Local therapy $(n=11)$} & Surgery & $\begin{array}{l}\text { Colon mass excision (1) } \\
\text { Axilla LN excision (1) }\end{array}$ & \\
\hline & \multirow{4}{*}{ Radiotherapy } & Abdomen LN (2) & \\
\hline & & Neck \& SCN LN (1) & \\
\hline & & Lung mass (2) & \\
\hline & & Bone (2) & \\
\hline & Gamma-knife surgery & Brain (2) & \\
\hline
\end{tabular}

Table 4. Subsequent therapy after progression. ICI immune checkpoint inhibitors.

A

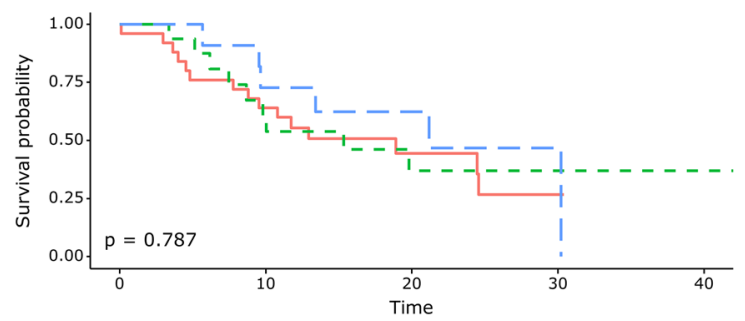

Number at risk

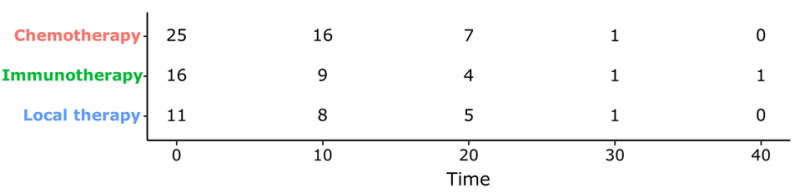

B

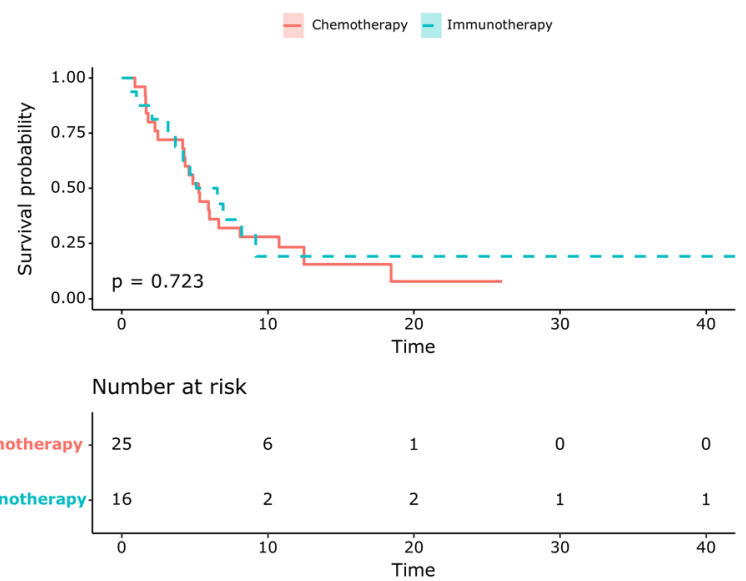

Figure 3. Kaplan-Meier survival curves showing (A) overall survival from the point of acquired resistance based on the subgroups of subsequent treatment (B) progression free survival based on the subsequent treatment.

Despite these limitations, our study described the clinical features and patterns of AR in detail. To our knowledge, this is the first study to show survival differences based on progression patterns. We believe that this report may provide useful information to clinicians regarding advanced NSCLC patients; additionally, it highlights the implications of subsequent therapy after AR.

In conclusion, advanced NSCLC patients who had durable responses ( $>6$ months) showed unique patterns of progression with high rates of progression among the LN only, in addition to oligo-progression. Local treatment and/or continuation of ICIs beyond AR might be an effective option for those patients. Further large-scale prospective studies are required to confirm these findings. 


\section{Methods}

Design, patients, and data collection. We retrospectively reviewed the records of patients diagnosed with NSCLC and treated with inhibitors of PD-1 and PD-L1 at the Seoul National University Hospital (SNUH) and Seoul National University Bundang Hospital (SNUBH) between January 1, 2011 and November 31, 2018.

Adults without a previous history of severe systemic disease or autoimmune diseases who received PD-1/ PD-L1 blockade alone or with cytotoxic T-lymphocyte associated protein 4 (CTLA-4) inhibitor or chemotherapy over the course of disease were included.

Patient classification and outcome measures. The patients were classified into the following subgroups: those who had intrathoracic, extrathoracic [excluding the liver], and liver progression. AR after durable response was defined as disease progression after 6 months with the best response of the stable disease, partial response, or complete response according to the Response Evaluation Criteria in Solid Tumors (RECIST) v1.1 criterion. Imaging studies included Computed tomography scan and Magnetic resonance imaging and were independently reviewed by two observers. Progression-free survival (PFS) was calculated from the ICI treatment initiation date to the date of disease progression using the RECIST v1.1 criteria $^{24}$, as confirmed by imaging, death, or the last follow-up date, if censored. OS was measured from the initiation of ICI treatment until death or the last follow-up date, if censored.

Statistical analyses. Continuous variables were compared using the Students t-test or the Mann-Whitney U-test, whereas categorical variables were compared using the chi-squared or Fisher exact tests. Survival analyses were conducted according to the Kaplan-Meier method using the log-rank test. All tests were two-sided and $p$-values $<0.05$ were considered to be statistically significant. For survival analysis, $\mathrm{R}$ version 3.4 .3 software $(\mathrm{R}$ Development Core Team, https://www.r-project.org/) was used for computation.

Ethics. All the included patients provided written, informed consent for data collection before treatment. The research team conducted retrospective analyses on cohort data. The institutional review boards of SNUH and SNUBH approved the study protocol (IRB number: B-2004-606-105). All procedures were carried out in accordance with the ethical standards of the institutional research ethics committee and the Helsinki declaration revised in 2013 by the World Medical Association.

Received: 12 July 2020; Accepted: 11 January 2021

Published online: 28 January 2021

\section{References}

1. Garon, E. B. et al. Pembrolizumab for the treatment of non-small-cell lung cancer. N. Engl. J. Med. 372, 2018-2028. https://doi. org/10.1056/NEJMoa1501824 (2015).

2. Gettinger, S. N. et al. Overall survival and long-term safety of nivolumab (anti-programmed death 1 antibody, BMS-936558, ONO-4538) in patients with previously treated advanced non-small-cell lung cancer. J. Clin. Oncol. 33, 2004-2012. https://doi. org/10.1200/jco.2014.58.3708 (2015).

3. Borghaei, H. et al. Nivolumab versus docetaxel in advanced nonsquamous non-small-cell lung cancer. N. Engl. J. Med. 373, 1627-1639. https://doi.org/10.1056/NEJMoa1507643 (2015).

4. Brahmer, J. et al. Nivolumab versus docetaxel in advanced squamous-cell non-small-cell lung cancer. N. Engl. J. Med. 373, 123-135. https://doi.org/10.1056/NEJMoa1504627 (2015).

5. Rittmeyer, A. et al. Atezolizumab versus docetaxel in patients with previously treated non-small-cell lung cancer (OAK): a phase 3, open-label, multicentre randomised controlled trial. Lancet (London, England) 389, 255-265. https://doi.org/10.1016/s01406736(16)32517-x (2017).

6. Gettinger, S. et al. Five-year followup of nivolumab in previously treated advanced non-small-cell lung cancer: results from the CA209-003 study. J. Clin. Oncol. 36, 1675-1684. https://doi.org/10.1200/jco.2017.77.0412 (2018).

7. Hellmann, M. D. et al. Estimating long-term survival of PD-L1-expressing, previously treated, non-small cell lung cancer patients who received pembrolizumab in KEYNOTE-001 and -010. J. Clin. Oncol. 35, 77-77. https://doi.org/10.1200/JCO.2017.35.7_suppl. 77 (2017).

8. Gettinger, S. et al. Five-year follow-up of nivolumab in previously treated advanced non-small-cell lung cancer: results from the CA209-003 study. J. Clin. Oncol. 36, 1675-1684. https://doi.org/10.1200/jco.2017.77.0412 (2018).

9. Yu, H. A. et al. Analysis of tumor specimens at the time of acquired resistance to EGFR-TKI therapy in 155 patients with EGFRmutant lung cancers. Clin. Cancer Res. Off. J. Am. Assoc. Cancer Res. 19, 2240-2247. https://doi.org/10.1158/1078-0432.Ccr-12-2246 (2013).

10. Anagnostou, V. et al. Evolution of neoantigen landscape during immune checkpoint blockade in non-small cell lung cancer. Cancer Discov. 7, 264-276. https://doi.org/10.1158/2159-8290.Cd-16-0828 (2017).

11. Gettinger, S. et al. Impaired hla class i antigen processing and presentation as a mechanism of acquired resistance to immune checkpoint inhibitors in lung cancer. Cancer Discov. 7, 1420-1435. https://doi.org/10.1158/2159-8290.cd-17-0593 (2017).

12. Zaretsky, J. M. et al. Mutations associated with acquired resistance to PD-1 blockade in melanoma. N. Engl. J. Med. 375, 819-829. https://doi.org/10.1056/NEJMoa1604958 (2016).

13. Gettinger, S. N. et al. Clinical features and management of acquired resistance to PD-1 axis inhibitors in 26 patients with advanced non-small cell lung cancer. J. Thorac. Oncol. 13, 831-839. https://doi.org/10.1016/j.jtho.2018.03.008 (2018).

14. Shah, S. et al. Clinical and molecular features of innate and acquired resistance to anti-PD-1/PD-L1 therapy in lung cancer. Oncotarget 9, 4375-4384. https://doi.org/10.18632/oncotarget.23315 (2018).

15. Chen, D. S. \& Mellman, I. Oncology meets immunology: the cancer-immunity cycle. Immunity 39, 1-10. https://doi.org/10.1016/j. immuni.2013.07.012 (2013).

16. Friedrich, M. et al. Tumor-induced escape mechanisms and their association with resistance to checkpoint inhibitor therapy. Cancer Immunol. Immunother. 68, 1689-1700. https://doi.org/10.1007/s00262-019-02373-1 (2019). 
17. Gandara, D. R. et al. Atezolizumab treatment beyond progression in advanced NSCLC: results from the randomized, phase III OAK study. J. Thorac. Oncol. 13, 1906-1918. https://doi.org/10.1016/j.jtho.2018.08.2027 (2018).

18. Long, G. V. et al. Nivolumab for patients with advanced melanoma treated beyond progression: analysis of 2 phase 3 clinical trials. JAMA Oncol. 3, 1511-1519. https://doi.org/10.1001/jamaoncol.2017.1588\%JJAMAOncology (2017).

19. Beaver, J. A. et al. Patients with melanoma treated with an anti-PD-1 antibody beyond RECIST progression: a US Food and Drug Administration pooled analysis. Lancet Oncol. 19, 229-239. https://doi.org/10.1016/S1470-2045(17)30846-X (2018).

20. Kazandjian, D., Keegan, P., Suzman, D. L., Pazdur, R. \& Blumenthal, G. M. Characterization of outcomes in patients with metastatic non-small cell lung cancer treated with programmed cell death protein 1 inhibitors past RECIST version 1.1-defined disease progression in clinical trials. Semin. Oncol. 44, 3-7. https://doi.org/10.1053/j.seminoncol.2017.01.001 (2017).

21. Necchi, A. et al. Atezolizumab in platinum-treated locally advanced or metastatic urothelial carcinoma: post-progression outcomes from the phase II IMvigor210 study. Ann. Oncol. 28, 3044-3050. https://doi.org/10.1093/annonc/mdx518 (2017)

22. George, S. et al. Safety and efficacy of nivolumab in patients with metastatic renal cell carcinoma treated beyond progression: a subgroup analysis of a randomized clinical trial. JAMA Oncol. 2, 1179-1186. https://doi.org/10.1001/jamaoncol.2016.0775\%JJAMA Oncology (2016).

23. Adam, L. C. et al. Cryotherapy for nodal metastasis in NSCLC with acquired resistance to immunotherapy. J. Immunother. Cancer 6, 147. https://doi.org/10.1186/s40425-018-0468-x (2018)

24. Eisenhauer, E. A. et al. New response evaluation criteria in solid tumours: revised RECIST guideline (version 1.1). Eur. J. Cancer (Oxford, England: 1990) 45, 228-247. https://doi.org/10.1016/j.ejca.2008.10.026 (2009).

\section{Acknowledgements}

This study was supported by the Grants from the SNUBH Research Fund (Grant Number: 13-2017-002 to SH Kim).

\section{Author contributions}

Writing: J.Y.H. Data acquisition: J.Y.H and S.H.Y, Supervision and review: C.O, M.K., B.K., T.M.K., D.K., D.S.H , K.J.S., S.H.K., Y.J.K. and J.S.L. Editing: J.Y.H. and S.H.K.

\section{Competing interests}

The authors declare no competing interests.

\section{Additional information}

Supplementary Information The online version contains supplementary material availlable at https://doi.org/ 10.1038/s41598-021-81666-x.

Correspondence and requests for materials should be addressed to S.H.K.

Reprints and permissions information is available at www.nature.com/reprints.

Publisher's note Springer Nature remains neutral with regard to jurisdictional claims in published maps and institutional affiliations.

(c) (i) Open Access This article is licensed under a Creative Commons Attribution 4.0 International License, which permits use, sharing, adaptation, distribution and reproduction in any medium or format, as long as you give appropriate credit to the original author(s) and the source, provide a link to the Creative Commons licence, and indicate if changes were made. The images or other third party material in this article are included in the article's Creative Commons licence, unless indicated otherwise in a credit line to the material. If material is not included in the article's Creative Commons licence and your intended use is not permitted by statutory regulation or exceeds the permitted use, you will need to obtain permission directly from the copyright holder. To view a copy of this licence, visit http://creativecommons.org/licenses/by/4.0/.

(C) The Author(s) 2021 